\title{
Protective role of interleukin-18 against Fas-mediated liver injury
}

\author{
NORIHIKO YAMAMOTO ${ }^{1}$, KAZUMOTO MURATA $^{2}$, KENTARO YONEDA $^{1}$, HIROYUKI FUKE $^{1}$, \\ YUMI YAMAGUCHI ${ }^{1}$, KEIICHI ITO ${ }^{1}$, KAZUSHI SUGIMOTO ${ }^{1}$, KATSUYA SHIRAKI ${ }^{1}$, \\ KEI-ICHI YAMANAKA ${ }^{3}$, HITOSHI MIZUTANI ${ }^{3}$ and YOSHIYUKI TAKEI ${ }^{1}$ \\ ${ }^{1}$ Department of Gastroenterology, Mie University Graduate School of Medicine, Tsu City, Mie Prefecture; \\ ${ }^{2}$ Center for Community Medicine, Jichi Medical University, Shimotsuke, Tochigi Prefecture; ${ }^{3}$ Department \\ of Dermatology, Mie University Graduate School of Medicine, Tsu City, Mie Prefecture, Japan
}

Received February 19, 2008; Accepted April 2, 2008

\begin{abstract}
Interleukin (IL)-18 plays an important role in the pathogenesis of several liver diseases as well as Fas-mediated apoptosis. However, the effects of IL-18 on Fas-mediated liver injury have not been well elucidated. Therefore, we examined the effects of IL-18 on Fas-mediated apoptosis in in vitro and in vivo experiments. We found that recombinant IL-18 protected mouse hepatocellular carcinoma cell lines, BNL5, from Fas-mediated apoptosis in a dose-dependent manner with up-regulation of both nuclear factor (NF) $\kappa B$ and $\mathrm{X}$-linked inhibitors of apoptosis (XIAP). IL-18 transgenic (Tg) mice were also protected from Fas-mediated liver injury and this was further confirmed by histological study and TUNEL staining. In IL-18 Tg mice, up-regulation of XIAP and downregulation of caspase 3 were observed after injection of antiFas, which was consistent with the in vitro findings. These results suggest that IL-18 suppresses Fas-mediated apoptosis of hepatocytes by up-regulation of NFKB and XIAP, following inhibition of caspase- 3 activity. This observation raises the possibility that IL-18 could be a therapeutic strategy for Fasmediated liver injury as a negative regulator of XIAP.
\end{abstract}

\section{Introduction}

Interleukin (IL)-18 is a unique cytokine which has biphasic function in immune response. IL-18 stimulates Th1-mediated immune response, which plays a critical role in the host defense against infection with intracellular microbes (1). IL-18 augments NK activity through the induction of constitutively expressed IL-18 receptor on NK cells (2). IL-18 inhibited

Correspondence to: Dr Kazumoto Murata, Center for Community Medicine, Jichi Medical University, 3311-1 Yakushiji, Shimotsuke, Tochigi 329-0498, Japan

E-mail: atarum@jichi.ac.jp

Key words: interleukin-18, Fas-mediated apoptosis, nuclear factor $\kappa \mathrm{B}, \mathrm{X}$-linked inhibitors of apoptosis
HBV replication by IFN- $\gamma$ production from intrahepatic NK and NKT cells (3). IL-18 also exhibited a major protective role in mice models of herpes simplex infection (4) or vaccinia virus infection (5). On the other hand, IL-18 induced naïve $\mathrm{T}$ cells to develop into Th2 cells in combination with IL-4 (6). With respect to the above, discussion focused mainly on the immunological roles of IL-18. Clinically, IL-18 is involved in several liver diseases including fulminant hepatitis, viral liver cirrhosis and primary biliary cirrhosis. In patients with these conditions, the level of serum IL-18 correlated with the severity of the disease (7) although IL-18 itself was not cytotoxic against hepatocytes $(8,9)$. However, the effects of IL-18 on Fas-mediated liver injury have not been fully elucidated.

Fas-mediated apoptosis has been implicated in several liver diseases as hepatocytes constitutively express Fas and are highly sensitive to stimulation on Fas receptor $(10,11)$. Since IL-18 is a potent inducer of NFkB (12), which acts as an anti-apoptotic factor, IL-18 may affect apoptosis to some extent. IL-1ß, which has a similar biological function to IL-18, protected mice from Fas-mediated liver injury through the suppression of caspase-3-like activity (13). Furthermore, recombinant IL-18 suppressed etoposide-induced apoptosis in HCC cells (14). From this evidence, we hypothesized that IL-18 has a potential protective mechanism against Fasmediated liver injury.

In the current study, we demonstrated that Fas-mediated apoptosis was significantly inhibited in IL-18 transgenic (Tg) mice in comparison with $\mathrm{C} 57 \mathrm{BL} / 6$ mice. Furthermore, we found that the anti-apoptosis mechanism of IL-18 appears to be up-regulation of X-linked inhibitors of apoptosis (XIAP) through up-regulation of $\mathrm{NF \kappa B}$.

\section{Materials and methods}

Cell line. A mouse hepatocellular carcinoma cell line, BNL5, was cultured in Dulbecco's modified Eagle's medium (DMEM) with $10 \%$ fetal calf serum.

Animals. Male C57BL/6 mice (8-12 wks) were purchased from SLC (Shizuoka, Japan) and maintained under conditions of controlled temperature and light with free access to food 
and water. The IL-18 Tg mouse is a keratin-14 promoter driven IL-18 transgenic mouse that constitutively secretes a mature form of IL-18 in the epidermis. IL-18 Tg show marked lichenification, and severe itchy dermatitis along with massive skin infiltration of lymphocytes, mast cells, and neutrophils. Plasma IL-18, IgE, and histamine levels are markedly elevated and thus they are considered mouse models of atopic dermatitis. Skin irritation along with severe scratching is commonly observed after the age of 6 months under specific pathogen-free (SPF) conditions without differences among mice (6). Age- and sex-matched C57/BL6 mice were used as control animals. IL-18 Tg mice were backcrossed against C57BL/6 mice more than 10 generations. All animals received humane care according to the institute's guidelines. For Fas-mediated liver injury, mice were intravenously injected with $0.25 \mu \mathrm{g} /$ body weight $(\mathrm{g})$ of antiFas (anti-CD95) (BD Bioscience, San Jose, CA) in $200 \mu 1$ of PBS. Blood samples were taken by retro-orbital puncture $6 \mathrm{~h}$ after injection.

Reagents. Anti-cleaved caspase-3 (Asp175) (Rand D Systems, Inc., Minneapolis, MN), anti-human IAP-like protein (hILP)/X-linked inhibitor of apoptosis protein (XIAP), FITCanti-Fas and FITC-anti-immunoglobulin $\mathrm{G}$ were purchased from BD Bioscience. FITC-anti-Fas ligand was purchased from Medical and Biological Laboratories Co. (Nagoya, Japan). Recombinant mouse IL-18 was purchased from MBL (Nagoya, Japan).

Detection of apoptosis. To assess viability of the BNL5 cells, 3-(4,5-dimethylthiazol-2-yl)-2,5-diphenyl tetrazolium bromide (MTT) assay was performed. BNL5 cells were seeded at a density of $1 \times 10^{4}$ cells/well in a 96-well microtiter plate (Corning Glass Works, Corning, NY). After confluence, the medium was replaced with various concentrations of recombinant IL-18 (rIL-18) in $50 \mu 1$ of 10\% DMEM. Thirty minutes later, $50 \mu 1$ of $10 \%$ DMEM with anti-CD95 (100 ng/ml) was added to each well and the plates were incubated for another $24 \mathrm{~h}$.

Activity of $N F \kappa B$. Confluent BNL5 cells in the $15-\mathrm{cm}$ plates were treated with $10 \mathrm{ng} / \mathrm{ml}$ of rIL-18 at various time points. For another experiment, confluent BNL5 cells were incubated with various concentrations of rIL-18 for $1 \mathrm{~h}$. Nuclear extracts of cells were obtained by mini-nuclear extract protocol as previously reported (15). NFKB activity in the nucleus was determined by NFKB Transfer Kit (Clontech, CA) according to the manufacturer's protocol.

Biochemical analysis. Serum alanine aminotransferase was measured by a standard method.

Detection of serum IL-18. The level of IL-18 in the serum was determined by an enzyme-linked immunosorbent assay (ELISA) kit (MBL, Nagoya, Japan) according to the manufacturer's protocol.

Histological examination. Liver samples were fixed with $10 \%$ formaldehyde and embedded in paraffin, sectioned, and stained with hematoxylin and eosin. Nuclear DNA fragmen- tation was detected by the ApopTag In Situ Apoptosis Detection Kit (Chemicon International, Temecula, CA) according to the manufacturer's protocol [terminal deoxynucleotidyl transferase-mediated deoxyuridine triphosphate biotin nick end-labeling (TUNEL) staining]. Briefly, the sections were deparaffinized and digested by proteinase $\mathrm{K}$ followed by inactivation of endogenous peroxidase with $3.0 \%$ hydrogen peroxide. The sections were incubated with TdT enzyme in a humidified chamber at $37^{\circ} \mathrm{C}$ for $1 \mathrm{~h}$. After washing, they were incubated with anti-digoxygenin coupled to peroxidase. Cells with nuclear DNA fragmentation were identified after incubating the sections with 3,3'-diaminobenzene (DAB) and $\mathrm{H}_{2} \mathrm{O}_{2}$. The sections were counterstained with $0.5 \%$ methyl green.

Western blotting. Mice were sacrificed $6 \mathrm{~h}$ after injection of anti-Fas antibody. Livers were immediately removed and stocked at $-80^{\circ} \mathrm{C}$ until use. Liver tissues were powdered using a cryopress and then dissolved with $500 \mu 1$ of radio-immunoprecipitation assay (RIPA) buffer. Each extract was separated by $10 \%$ sodium dodecyl sulfate-polyacrylamide gradient gel electrophoresis (SDS-PAGE) and transferred onto nitrocellulose membranes. After blocking, the membrane was incubated with anti-cleaved caspase-3 $(1: 1,000)$ or anti-hILP/XIAP (1:500) at $4{ }^{\circ} \mathrm{C}$ overnight. Anti- $\alpha$-tubulin (Calbiochem, San Diego, CA) was used as an internal control. The proteins were detected using electrochemiluminescence techniques (Pierce Chemicals, Rockford, IL).

The expression of FAS and FasL on hepatocytes. Livers from C57BL/6 or IL-18 Tg mice were pressed through a $70-\mu \mathrm{m}$ cell strainer (Becton Dickinson, $\mathrm{NJ}$ ) and washed once with ice-cold RPMI. Hepatocytes were obtained by centrifugation at $420 \mathrm{rpm}$ for $5 \mathrm{~min}$ and were subject to Fas or FasL staining (FITC-conjugated anti-Fas or FasL antibody, BD Bioscience). All analyses were performed on a fluorescence-activity cell sorter (FACS) caliber cytometer (Becton Dickinson).

Statistical analysis. Statistical analysis was performed by Student's t-test. P-values less than 0.05 were considered significant.

\section{Results}

Recombinant IL-18 protects BNL5 cells from Fas-mediated apoptosis. Fas-mediated apoptosis in BNL5 cells was inhibited by rIL-18 in a dose-dependent manner (Fig. 1A). On the other hand, $10 \mathrm{ng} / \mathrm{ml} \mathrm{rIL}-18$ enhanced NFKB in the BNL5 cells, and NFKB in the nucleus showed a peak at $0.5 \mathrm{~h}$ after administration (Fig. 1B). NFKB in the BNL5 cells increased in a dose-dependent manner and showed a peak at $10 \mathrm{ng} / \mathrm{ml}$ of rIL-18 (Fig. 1C). XIAP expression was increased by rIL-18 and showed a peak at $10 \mathrm{ng} / \mathrm{ml}$ of rIL-18 (Fig. 1D). Thus, rIL-18 protected BNL5 cells from apoptosis and upregulated NFKB and XIAP expression in the BNL5 cells.

Inhibition of Fas-mediated liver injury in IL-18 Tg mice. We attempted to ascertain whether IL-18 Tg mice were protected from Fas-mediated apoptosis. First, we confirmed that serum IL-18 levels in IL-18 Tg were significantly higher 

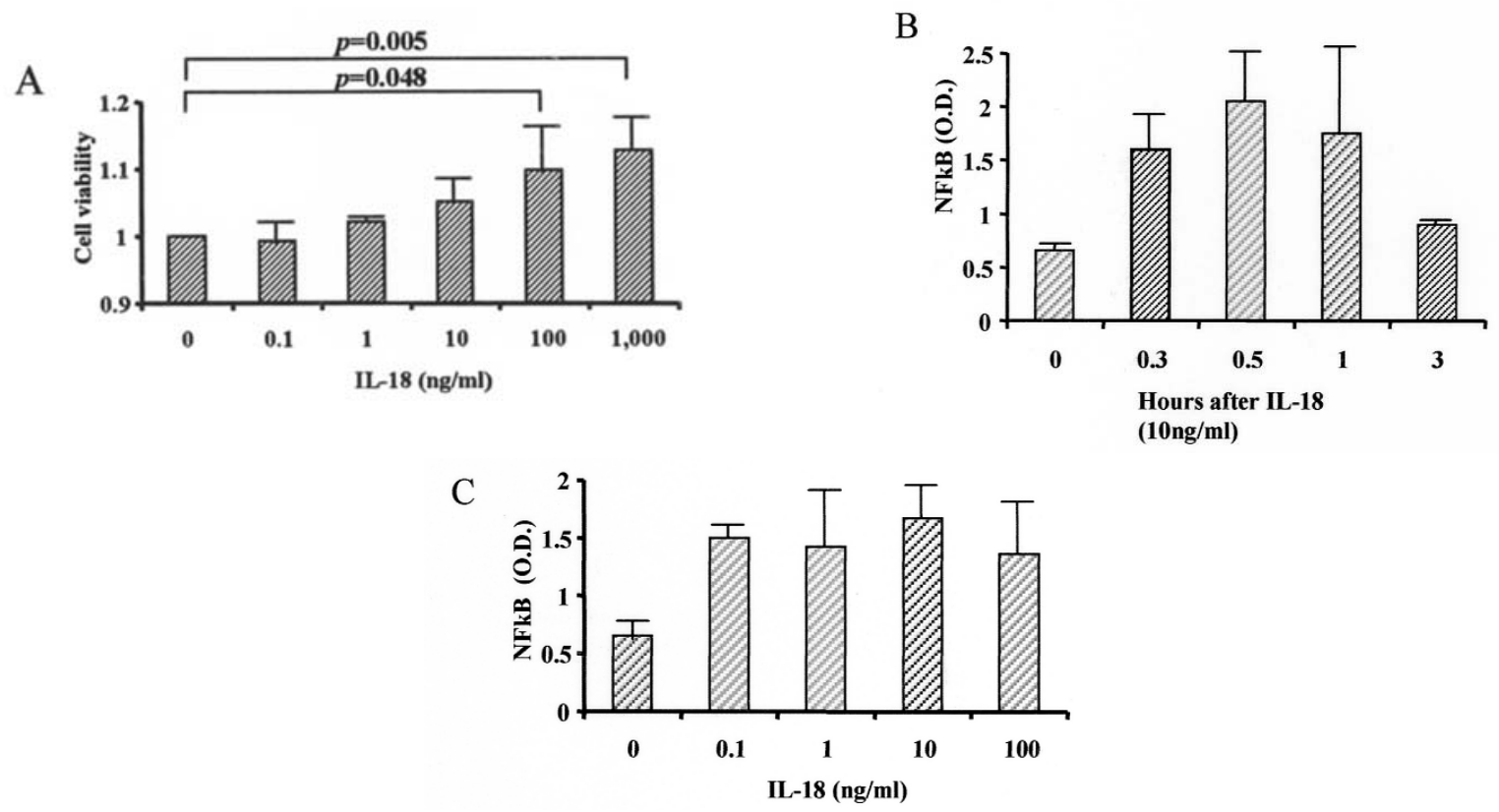

D

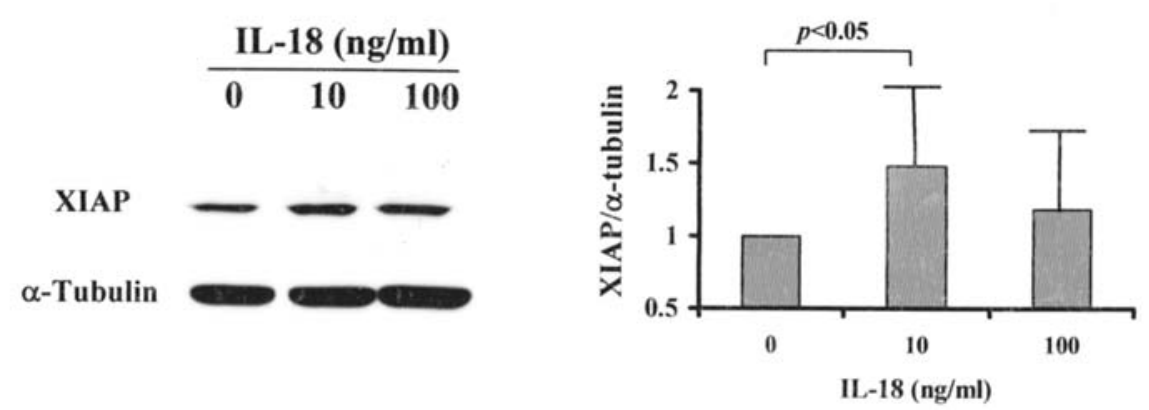

Figure 1. (A) MTT assay. BNL5 cells were protected from Fas-mediated apoptosis by rIL-18 in a dose-dependent manner. (B) NFkB levels in BNL5 cells showed a peak at $0.5 \mathrm{~h}$ after rIL-18 treatment $(10 \mathrm{ng} / \mathrm{ml})$. (C) NFKB levels in BNL5 cells showed a peak at $10 \mathrm{ng} / \mathrm{ml}, 0.5 \mathrm{hour}$ after rIL-18 treatment. (D) XIAP expression was up-regulated by rIL-18 and showed a peak at $10 \mathrm{ng} / \mathrm{ml}$ after rIL-18 treatment.

than those in the C57BL/6 mice before anti-Fas treatment (Fig. 2A). Six hours after injection of anti-Fas, serum ALT levels were markedly increased in C57BL/6 mice whereas serum ALT levels were significantly lower in the IL-18 Tg mice (Fig. 2B). These differences were also observable in the gross appearance of the liver surface with the liver color turning dark red and evident swelling in the C57BL/6 mice, whereas these changes were mild in IL-18 Tg mice (Fig. 2C). The changes were also confirmed by histological examination. Gross hemorrhagic hepatic necrosis was observed in the C57BL/6 mice (Fig. 2Da) whereas it was much less evident in the IL-18 Tg (Fig. 2Db). These differences were further corroborated by TUNEL staining (Fig. 2Dc and d). The number of TUNEL-positive cells was significantly lower in the IL-18 Tg mice than in the C57BL/6 mice (Fig. 2E). These results indicate that Fas-mediated apoptosis in the liver was significantly inhibited in the IL-18 Tg mice in comparison with the C57BL/6 mice.

Fas and Fas ligand (FasL) expression on hepatocytes. As shown in Fig. 3, the expression levels of both Fas and FasL on hepatocytes were similar between the IL-18 Tg and the C57BL/6 mice. The Fas or FasL expression levels may not contribute to the reduction of liver injury in IL-18 Tg mice.
Caspase-3 cleavage and XIAP in a Fas-mediated liver injury model. Caspase- 3 is one of the best characterized proteases downstream of Fas stimulations. Therefore, we examined caspase- 3 activity $6 \mathrm{~h}$ after anti-Fas injection. The expression level of cleaved caspase- 3 was significantly reduced in the IL-18 Tg mice in comparison with the C57BL/6 mice (Fig. 4). Next, we tried to determine which apoptotic pathways were impaired in the IL-18 Tg mice. The expression level of XIAP was significantly up-regulated in the IL-18 Tg mice in comparison with that in the C57BL/6 mice. Collectively, these results suggested that the suppressive effect of Fasinduced liver injury in the IL-18 Tg mice was regulated through direct caspase- 3 inhibition by XIAP. These observations were consistent with in vitro experiments.

\section{Discussion}

In this study, we examined the effects of IL-18 on Fas-mediated apoptosis. We found that IL-18 Tg mice were protected from Fas-mediated liver injury through up-regulation of the anti-apoptotic pathway, and this was further confirmed by in vitro experiments.

IL-18 plays an important role in immunological response and is related to various liver diseases. In this study, we 

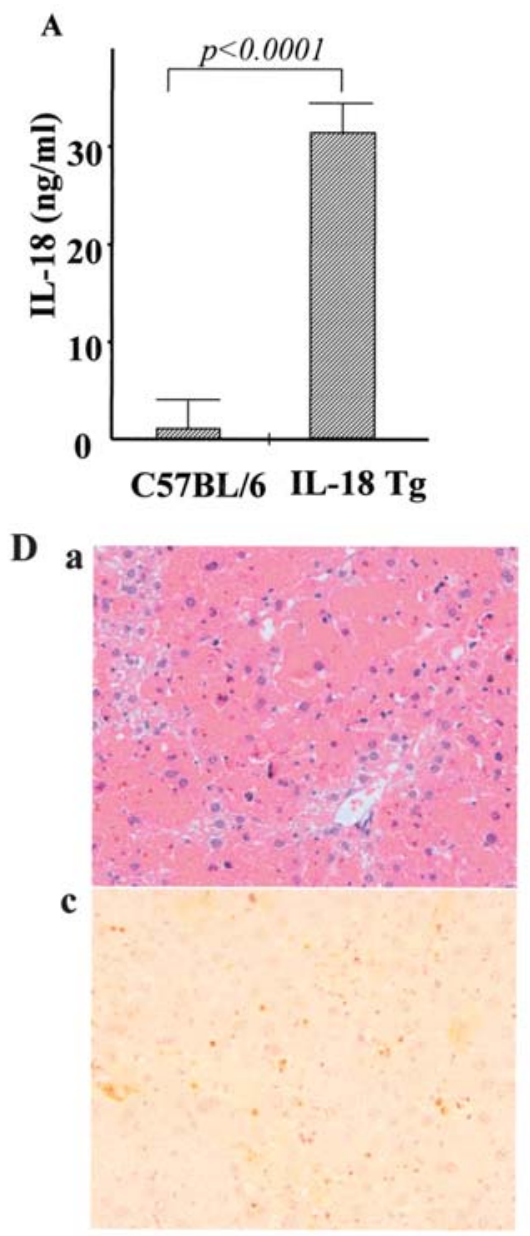

C57BL/6
B
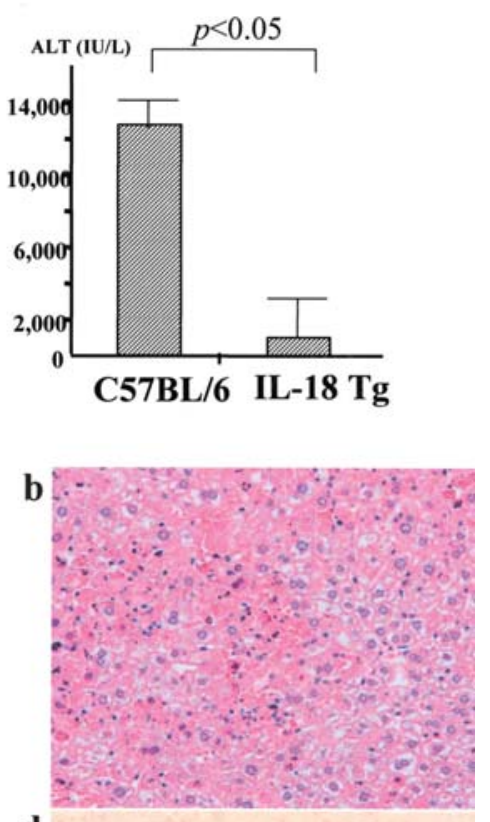

d

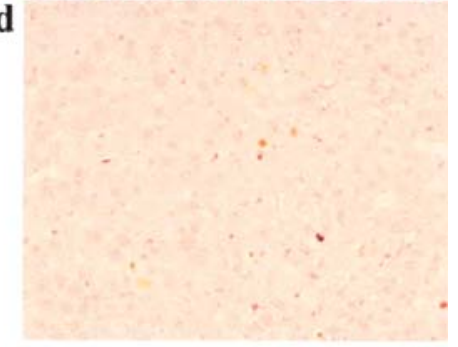

IL-18Tg
C

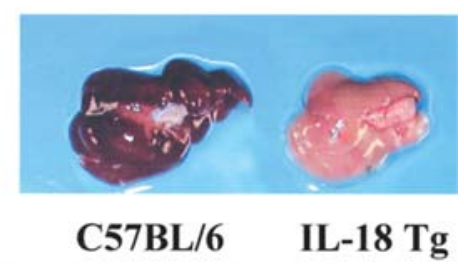

E

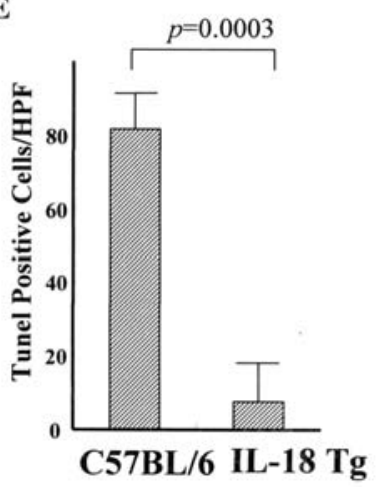

Figure 2. (A) Serum IL-18 levels were significantly higher in IL-18 Tg mice than in C57BL/6 mice ( $<<0.0001)$. (B) Serum ALT levels after injection of anti-Fas were significantly lower in IL-18 Tg mice than in C57BL/6 mice $(\mathrm{p}<0.05)$. (C) Gross appearance of liver in IL-18 Tg and C57BL/6 mice after injection of anti-Fas antibody. Liver color turned dark red and swelling was evident in the C57BL/6 mice whereas these changes were very mild in the IL-18 Tg mice. (D) Hematoxylin and eosin staining in the C57BL/6 (a) and the IL-18 Tg (b) mice after injection of anti-Fas. TUNEL staining in the C57BL/6 (c) and the IL-18 Tg (d) mice after injection of anti-Fas. (E) The number of TUNEL-positive cells was significantly lower in the IL-18 Tg mice than in the C57BL/6 mice.

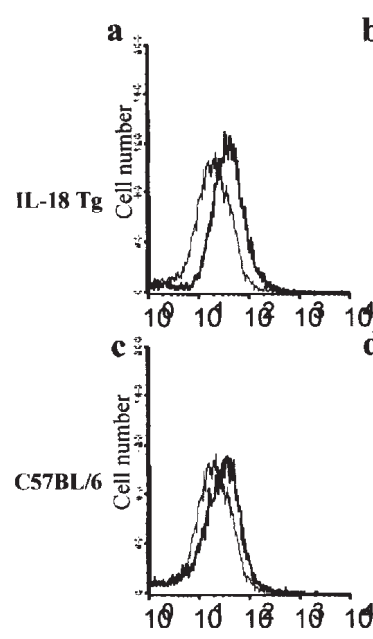

Fas

\section{Fluorescent intensity}

Figure 3. Fas and FasL expression on hepatocytes. The expression levels of both Fas and FasL on hepatocytes were similar in the IL-18 Tg and the C57BL/6 mice (IgG anti-Fas or anti FasL).

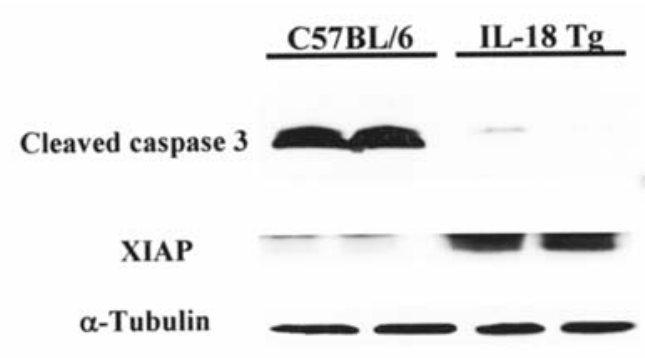

Figure 4. Apoptotic protease expression in C57BL/6 and IL-18 Tg mice after injection of anti-Fas. The expression level of cleaved caspase-3 was significantly reduced in IL-18 Tg mice with up-regulation of XIAP in comparison with the C57BL/6 mice (representative data of two mice from each group shown).

focused on IL-18 as an anti-apoptotic factor, and we examined the relation between IL-18 and Fas-mediated apoptosis. We demonstrated that rIL-18 inhibited the Fasmediated apoptosis of BNL5 cells in a dose- and timedependent manner. Furthermore, the expression of $\mathrm{NF \kappa B}$ and 
XIAP in BNL5 cells were up-regulated by rIL-18. These upregulations may result in the protection of BNL5 cells from apoptosis since they are upstream of caspase- 3 and downregulate caspase-3. Previous studies suggested that $\mathrm{NF \kappa B}$ regulated XIAP gene expression, resulting in protection from tumor necrosis factor (TNF) $\alpha$-induced apoptosis (16), which is consistent with our results. It is well known that the activation of NFKB is found to block the activation of caspase through the inhibitors of apoptosis (IAP) family (17). Therefore, we examined XIAP expression in our model. After injection of anti-Fas antibody in IL-18 Tg mice, Fas-mediated liver injury was remarkably suppressed relative to C57BL/6 mice. In addition, the expression level of cleaved caspase- 3 was significantly reduced in IL-18 Tg mice with up-regulation of XIAP in comparison with the C57BL/6 mice.

A previous study suggested that IL-18 Tg mice showed hepatocyte apoptosis without any treatment through spontaneous activation of the Fas pathway, as well as swollen livers (18). However, in our study, no ALT elevation was observed in IL-18 Tg mice without any treatment, and liver weight per body weight was similar to C57BL/6 mice (data not shown). On the contrary, anti-apoptotic effects against anti-Fas were observed in our IL-18 Tg mice. These differences may result from genetic differences between the two $\mathrm{Tg}$ mice. Serum IL-18 level in IL-18 Tg mice in the abovementioned study was half that in our IL-18 Tg mice, which may also be a factor.

In other studies, IL-18 up-regulated functional FasL expression in NK cells (19), T cells (20) and a human hepatocellular carcinoma cell line (21). In this case, the antiapoptotic effects in our study may result from up-regulation of FasL. Therefore, we analyzed Fas and FasL expression using hepatocytes from the C57BL/6 or the IL-18 Tg mice before any treatment. However, Fas or FasL expression on the hepatocytes was similar in C57BL/6 and IL-18 Tg mice.

NFkB has been shown to play an important role in the survival and regeneration of hepatocytes (22). NFkB knockout mice exhibit massive apoptosis of the hepatocyte, resulting in embryonic lethality (23). In this study, we demonstrated a dose-dependent and a time-dependent up-regulation of NFKB by recombinant IL-18 in the BNL5 cells. Furthermore, in the IL-18 Tg mice, NFкB expression was up-regulated compared to wild mice during hepatic injury. Since NFKB stimulates the expression of anti-apoptotic molecules, we examined XIAP expression. In the IL-18 Tg mice, the expression level of XIAP was up-regulated. Thus, in IL-18 Tg mice, Fasmediated liver injury was protected by inhibiting caspase- 3 activity. In HCC cell lines, NFkB activity and the expression of XIAP mRNA were increased by treatment with recombinant human IL-18 (14). Furthermore, in IL-18 Tg mice, the NFkB levels of hepatocytes were increased (18), which is consistent with our results. In this experimental model, the same mechanism may contribute to the inhibition of Fas-mediated liver injury in the IL-18 Tg mice. Anti-apoptotic effects of IL-1ß, which has a similar biological function to IL-18, in Fas-mediated liver injury (13) or anti-apoptotic effect of IL-18 in hepatocellular carcinoma (14) have previously been reported. These findings suggest the protective role of IL-18 against apoptosis and potential therapeutic use of IL-18 for several liver diseases.
On the other hand, IL-18 has also induced apoptosis in human cardiac microvascular endothelial cells (12). The action of IL-18 could thus be controversial or have organdependent mechanisms and this should be taken into account for clinical applications.

In conclusion, IL-18 has an anti-apoptotic effect through the up-regulation of $\mathrm{NF \kappa B}$, and subsequently suppresses $\mathrm{XIAP}$ and caspase- 3 evidenced by both in vitro and in vivo study. From our findings, IL-18 may serve as a negative regulator of apoptosis, and IL-18 itself or stimulators of the IL-18 pathway could be useful therapeutic options against several liver diseases in the future.

\section{References}

1. Sugihara I: Interleukin-18 (IL-18) and infectious disease, with special emphasis on disease induced by intracellular pathogen. Microbes Infect 2: 1257-1263, 2000.

2. Hyodo Y, Matsui K, Hayashi N, Tsutsui H, Kashiwamura S-I, Yamauchi H, Hiroshi K, Takeda K, Tagawa Y-I, Iwakura Y, Kayagaki N, Kurimoto M, Okamura H, Hada T, Yagita H, Akira S, Nakanishi K and Higashino K: IL-18 up-regulates perforin-mediated NK activity without increasing perforin messenger RNA expression by binding to constitutively expressed IL-18 receptor. J Immunol 162: 1662-1668,1999.

3. Kimura K, Kakimi K, Wieland S, Guidotti LG and Chisari FV: Interleukin-18 inhibits hepatitis B virus replication in the livers of transgenic mice. J Virol 76: 10702-10707, 2002.

4. Fujioka N, Akazawa R, Ohashi K, Ikeda M and Kurimoto M: Interleukin-18 protects mice against acute herpes simplex virus type 1 infection. J Virol 73: 2401-2409, 1998.

5. Tanaka-Kataoka M, Kunikata T, Takayama S, Iwaki K, Ohashi K, Ikeda $\mathrm{M}$ and Kurimoto $\mathrm{M}$ : In vivo antiviral effect of interleukin 18 in a mouse model of vaccinia virus infection. Cytokine 11: 593-599, 1999

6. Konishi H, Tsutsui H, Murakami T, et al: IL-18 contributes to the spontaneous development of atopic dermatitis-like inflammatory skin lesion independently of IgE/stat6 under specific pathogen-free conditions. Proc Natl Acad Sci USA 99: 11340-11345, 2002.

7. Tsutsui H, Adachi K, Seki E and Nakanishi K: Cytokine-induced inflammatory liver disease. Curr Molec Med 3: 545-559, 2003.

8. Tsutsui H, Kawagaki N, Kuida K, Nakano H, Hayashi N, Takeda K, Matsui K, Kashiwamura S, Hada T, Akira S, Yagita H, Okamura H and Nakanishi K: Caspase-1 independent, Fas/Fas ligand-mediated IL-18 secretion from macrophages causes acute liver injury in mice. Immunity 11: 359-367, 1999.

9. De la Barrera S, Finiasz M, Fink S, Ilarregui J, Aleman M, Olivares L, Franco MC, Pizzariello G and Del Carmen Sasiain M: NK cells modulate the cytotoxic activity generated by mycobacterium leprae-hsp65 in leprosy patients: role of IL-18 and IL-13. Clin Exp Immunol 135: 105-113, 2004.

10. Galle PR and Krammer PH: CD95-induced apoptosis in human liver disease. Semin Liver Dis 18: 141-151, 1998.

11. Nanji AA: Apoptosis and alcoholic liver disease. Semin Liver Dis 18: 187-190, 1998.

12. Chandrasekar B, Vemula K, Surabhi RM, et al: Activation of intrinsic and extrinsic proapoptotic signaling pathway in interleukin-18-mediated human cardiac endothelial cell death. J Biol Chem 279: 20221-20233, 2004.

13. Takehara T, Hayashi N, Tatsumi T, Kanto T, Mita E, Sasaki Y, Kasahara $\mathrm{A}$ and Hori M: Interleukin $1 \beta$ protects mice from Fasmediated hepatocyte apoptosis and death. Gastroenterology 117 : 661-668, 1999.

14. Asakawa M, Kono H, Amemiya H, Matsuda M, Suzuki T, Maki A and Fujii H: Role of interleukin-18 and its receptor in hepatocelllular carcinoma associated with hepatitis $\mathrm{C}$ virus infection. Int J Cancer 118: 564-570, 2005.

15. Schreiber E, Matthias P, Muller MM and Schaffer W: Rapid detection of octamer binding proteins with 'mini-extracts', prepared from a small number of cells. Nucleic Acids Res 17: 6419, 1989.

16. Stehlik C, Martin R, Kumabashiri I, Schmid JA, Binder BR and Lipp J: Nuclear factor (NF)- $\mathrm{B}$-regulated X-chromosomelinked IAP gene expression protects endothelial cells from tumor necrosis factor $\alpha$-induced apoptosis. JEM 188: 211-216, 1998. 
17. Wang CY, Mayo MW, Korneluk RG, Goeddel DV and Baldwin AS Jr: NF-kappa B antiapoptosis: induction of TRAF-1 and TRAF-2 and c-IAP1 and c-IAP2 to suppress caspase- 8 activation. Science 281: 1680-1683, 1998.

18. Finotto S, Siebler J, Hausding M, Schipp M, Wirtz S, Klein S, Protschka M, Doganci A, Lehr HA, Trautwein C, Khosravi-Fahr R, Strand D, Lohse A, Galle PR, Blessing M and Neurath MF: Severe hepatic injury in interleukin 18 (IL-18) transgenic mice: a key role for IL-18 in regulating hepatocyte apoptosis in vivo. Gut 53: 392-400, 2004.

19. Tsutsui H, Nakanishi K, Matsui K, Higashino K, Okumura H, Miyazawa $\mathrm{Y}$ and Kaneda K: IFN- $\gamma$-inducing factor up-regulates Fas ligand-mediated cytotoxic activity of murine natural killer cell clones. J Immunol 157: 3967-3973, 1996.

20. Dao T, Ohashi K, Kayano T, Kurimoto M and Okumura H: Interferon-gamma-inducing factor, a novel cytokine, enhances Fas ligand-mediated cytotoxicity of murine T helper 1 cells. Cell Immunol 173: 230-235, 1996.
21. Lee MO, Choi YH, Shin E-C, Kang HJ, Kim YM, Jeong SY, Seong JK, Yu DY, Cho H, Park JH and Kim SJ: Hepatitis B virus $X$ protein induced expression of interleukin 18 (IL-18): a potential mechanism for liver injury caused by hepatitis B virus (HBV) infection. J Hepatol 37: 380-386, 2002.

22. Iimuro Y, Nishimura T, Hellerbrand C, Behrus KE, Schoonhoven R, Grisham JW and Brenner DA: NF kappa B prevents apoptosis and liver dysfunction during liver degeneration. J Clin Invest 101: 802-811, 1998

23. Begg AA, Sha WC, Bronson RT, Ghosh S and Baltimore D: Embryonic lethality and liver degeneration in mice lacking the RelA component of NF kappa B. Nature 376: 167-170, 1995. 